\title{
Q-TWiST analysis of lapatinib combined with capecitabine for the treatment of metastatic breast cancer
}

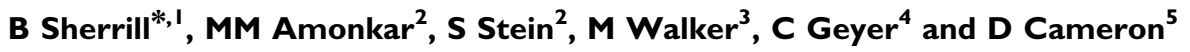 \\ ${ }^{\prime}$ RTI Health Solutions, Research Triangle Park, NC, USA; ${ }^{2}$ GlaxoSmithKline, Collegeville, PA, USA; ${ }^{3}$ GlaxoSmithKline, Greenford, UK; ${ }^{4}$ Allegheny Cancer \\ Center, Pittsburgh, PA, USA; ${ }^{5}$ University of Leeds, Leeds, UK
}

The addition of lapatinib (Tykerb/Tyverb) to capecitabine (Xeloda) delays disease progression more effectively than capecitabine monotherapy in women with previously treated HER2 + metastatic breast cancer (MBC). The quality-adjusted time without symptoms of disease or toxicity of treatment (Q-TWiST) method was used to compare treatments. The area under survival curves was partitioned into health states: toxicity (TOX), time without symptoms of disease progression or toxicity (TWiST), and relapse period until death or end of follow-up (REL). Average times spent in each state, weighted by utility, were derived and comparisons of Q-TWiST between groups performed with varying combinations of the utility weights. Utility weights of 0.5 for both TOX and REL, that is, counting 2 days of TOX or REL as I day of TWiST, resulted in a 7-week difference in quality-adjusted survival favouring combination therapy $(P=0.0013)$. The Q-TWiST difference is clinically meaningful and was statistically significant across an entire matrix of possible utility weights. Results were robust in sensitivity analyses. An analysis with utilities based on EQ-5D scores was consistent with the above findings. Combination therapy of lapatinib with capecitabine resulted in greater quality-adjusted survival than capecitabine monotherapy in trastuzumab-refractory MBC patients.

British Journal of Cancer (2008) 99, 7II-715. doi: I0.1038/sj.bjc.660450 I www.bjcancer.com

Published online 19 August 2008

(c) 2008 Cancer Research UK

Keywords: lapatinib; breast cancer; Q-TWiST; quality-adjusted survival

Lapatinib (Tykerb/Tyverb) combined with capecitabine (Xeloda) has been shown to significantly delay disease progression, as compared with capecitabine alone, in women with HER2 + $($ ErbB2 +$)$ metastatic breast cancer that has progressed after standard therapies of anthracyclines, taxanes and trastuzumab (Geyer et al, 2006; Cameron et al, 2008). The combination therapy of lapatinib with capecitabine resulted in similar types of adverse events (AEs), and no increase in serious toxic effects, as compared with capecitabine monotherapy. These previously reported analyses focus on efficacy and safety outcomes independently. In a cancer setting it becomes important to compare treatment risks and benefits within a single metric.

We therefore conducted a quality-adjusted time without symptoms of disease or toxicity of treatment (Q-TWiST) analysis, to better estimate the overall benefit for patients using a single metric that incorporates progression, survival, treatment toxicities and quality-of-life. Originally developed for evaluating breast cancer treatments, this quality-adjusted survival method describes both the quality and quantity of survival time using a single metric (Gelber and Goldhirsch, 1986; Gelber et al, 1991). Q-TWiST compares the relative therapeutic value of treatments based on the patient experience within the context of clinical outcomes related to cancer and its treatment. Thus, Q-TWiST provides a measure of clinical benefit not necessarily apparent from separate efficacy

*Correspondence: B Sherrill, RTI Health Solutions, 3040 Cornwallis Road, P O Box 12194, Research Triangle Park, NC 27709, USA; E-mail: bsherrill@rti.org

Received 26 March 2008; revised 16 May 2008; accepted 10 June 2008; published online 19 August 2008 and safety assessments. Assessments such as time to disease progression measure the length of time between clinical events but do not account for the value of that time to the patient.

The Q-TWiST method assumes that cancer patients progress through a set of health states of varying utility value for the individual patient. The health states most commonly used for Q-TWiST analyses of cancer treatment studies include: toxicity (TOX), time spent with toxic effects of study treatment; TWiST, time period without either symptoms of disease progression or toxicity; and relapse (REL), time following disease progression until death or end of follow-up (Cole et al, 2001).

Quality-adjusted survival is an understandable concept and can help individual patients make informed treatment decisions based on the relative importance they place on different health outcomes. Comparison of the amount of time a patient can expect before relapse without debilitating toxicities is a valuable aid in choosing between treatments. In this particular application, we were interested in showing whether patients on the combination of lapatinib and capecitabine would experience on average more time in a better health state compared with patients on capecitabine alone.

\section{MATERIALS AND METHODS}

\section{Data source}

The data source for this analysis was the previously reported Phase III clinical trial of lapatinib combined with capecitabine vs capecitabine alone in women with advanced or metastatic HER2 + breast cancer who had progressive disease following prior therapy which included an anthracycline, a taxane and trastuzumab 
(Geyer et al, 2006; Cameron et al, 2008). Subjects were randomised to receive either of the following treatments:

- Lapatinib $1250 \mathrm{mg}$ daily without interruption and capecitabine $2000 \mathrm{mg} \mathrm{m}^{-2}$ per day, days 1 through 14, every 21 days; or

- Capecitabine $2500 \mathrm{mg} \mathrm{m}^{-2}$ per day, days $1-14$, every 21 days.

Study enrollment was stopped early based on the unanimous recommendation of the independent data monitoring committee following a planned interim analysis. Based on independently reviewed imaging, the primary end point of time-to-progression had exceeded the predetermined stopping criteria, and review of toxicity data indicated no substantive safety concerns. The study closed to recruitment on 3 April 2006, when the results of the interim analysis were released and patients on monotherapy were offered the option of receiving the combination therapy. The analysis presented here uses data as of the lock date on 3 April 2006; thus, it does not take into account benefits that may have accrued beyond the end of the study.

\section{Definitions}

Toxicity In the primary analysis, the toxicity (TOX) state included all days spent with Grade 3/4 AEs after randomisation and prior to disease progression. A day with multiple AEs was only counted once. The TOX state comprised the total number of days spent with AEs, regardless of when AEs started or whether gaps occurred between AEs. Per convention, AEs occurring after progression were not included in the TOX state. Expanded definitions of the TOX state were examined in sensitivity analyses.

Progression-free survival The end of the time period without symptoms of toxicity or disease progression (TWiST) is based on progression-free survival (PFS), including events of disease progression and deaths due to any cause. For subjects who did not progress or die as of the last data date, PFS was censored at the time of the last independently assessed radiological scan preceding the initiation of any alternative anticancer therapy. If a subject had only a baseline visit or did not have an independently reviewed radiological scan dated prior to initiation of alternative anticancer therapy, PFS was censored at the date of randomisation.

Utility weights For the threshold utility analysis, a matrix of hypothetical utility weights $\left(u_{i}\right)$ for the TOX and REL health states was constructed by varying utility from 0 to 1 by 0.25 , resulting in 25 combinations, relative to the utility of TWiST. For treatment comparison purposes, TWiST is used as the reference state with utility set equal to 1 , representing the highest utility that can be expected for a patient with metastatic breast cancer.

Additionally, individual utilities were determined from patientreported health status on the EQ-5D (EuroQol Group, 1990), a simple and validated questionnaire measuring five dimensions: mobility, self-care, usual activities, pain/discomfort, and anxiety/ depression. The EQ-5D was scheduled to be completed predose on day 1 , every 6 weeks for the first 24 weeks, followed by every 12 weeks thereafter, and at withdrawal from the randomised therapy. Patient-reported utility weights were derived from the EQ-5D using published algorithms (AHRQ, 2005). The maximum possible value on the EQ5D is one; utility values less than zero represent a state evaluated as worse than death for the patient.

\section{Statistical analysis}

Estimation of health-state durations The Product-Limit Method (Kaplan and Meier, 1958) was used to estimate the mean amount of time in the following states:

- With toxicities after randomisation but prior to progression (i.e., TOX),
- From randomisation to progression or death (i.e., PFS), and - From randomisation until death from any cause (i.e., overall survival (OS)).

Survival curves corresponding to toxicity duration, PFS, and OS were plotted on a single graph for each treatment group. The areas between the curves represent the restricted mean durations of TWiST and REL as follows:

Duration of TWiST $=$ PFS time - time with toxicities; and

$$
\text { Duration of REL }=\text { OS time }- \text { PFS time }
$$

All patients in this study had previously relapsed, so the REL state here refers to the period after further progression. To look over the same timeframe for both treatments, analyses were restricted to the median overall follow-up time. This convention is required as analyses represent areas under the curve; both treatments are evaluated over the same period of time. The primary analysis was performed on the intent-to-treat (ITT) population, with TOX defined to include Grade 3/4 AEs. The results of this analysis are reported unadjusted and then are employed during the Q-TWiST calculation.

Calculation of Q-TWiST and threshold utility analysis Q-TWiST for each treatment arm was calculated as follows:

$$
\mathrm{Q}-\mathrm{TWiST}=\left(u_{\mathrm{TOX}} \times \mathrm{TOX}\right)+\mathrm{TWiST}+\left(u_{\mathrm{REL}} \times \mathrm{REL}\right)
$$

where TOX, TWiST, and REL represent the mean estimated healthstate durations, and $u_{\mathrm{TOX}}$ and $u_{\mathrm{REL}}$ denote utility weights for the TOX and REL states, respectively. Note that Q-TWiST equals the mean OS when $u_{\mathrm{TOX}}=u_{\mathrm{REL}}=1$ and equals the mean PFS when $u_{\mathrm{TOX}}=1$ and $u_{\mathrm{REL}}=0$.

A threshold utility analysis was performed to illustrate which utility combinations are expected to result in different durations of quality-adjusted survival between treatment groups. Differences in mean Q-TWiST between treatment groups were calculated for each combination of hypothetical utility weights (Cole et al, 2001). Ninety-five percent confidence intervals for the mean differences and two-sided $P$-values for testing the null hypothesis of no difference were performed, based on the normal approximation, with s.e. calculated by bootstrapping (Glasziou et al, 1990). Points between selected utility values can be interpolated from the threshold utility graph.

Sensitivity analyses In two sensitivity analyses, the TOX state was redefined to include (1) all AEs of any grade or (2) AEs of any grade classified as treatment-related according to the protocol.

Incorporation of observed utility data For each patient, the average utility weight derived from EQ-5D assessments during a health state was assigned as a per-person utility weight for the TOX, TWiST, and REL states. If a utility weight for the TWiST state was not available for a patient, then the predose EQ-5D score was used. For patients who progressed (i.e., were not censored for progression), the last EQ-5D score after progression was used for the REL state. Patients who died on the date of progression were assigned utility $=0$ for the REL state. To compare groups, the overall average utility relative to the average reported utility for the TWiST state was used.

\section{RESULTS}

As of 3 April 2006, 399 patients were enrolled and randomised either to lapatinib plus capecitabine $(n=198)$ or to capecitabine alone $(n=201)$. The ITT population includes all randomised patients, who were well balanced across treatment groups. All 


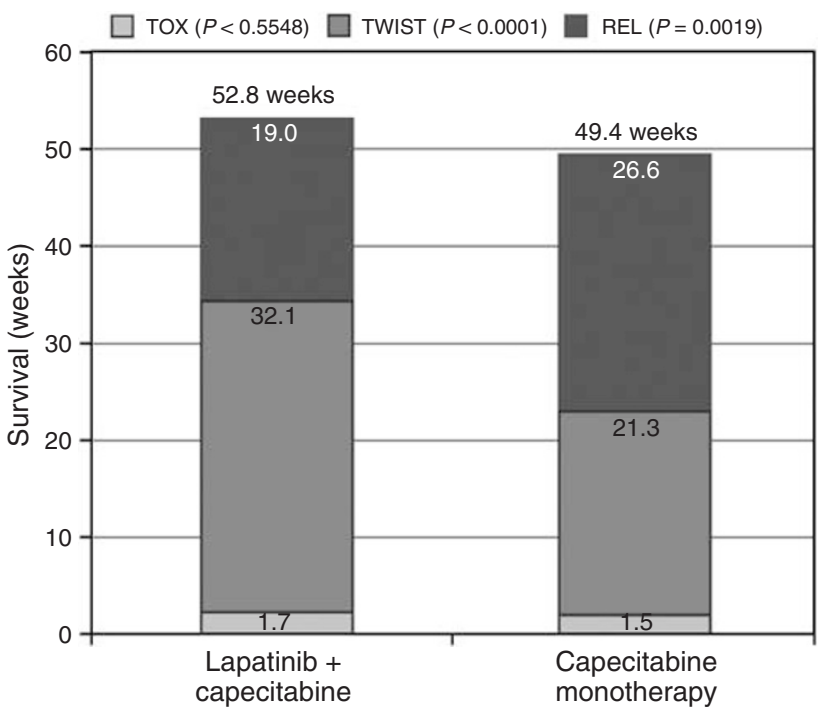

Figure I Unweighted mean duration of health states (weeks). TOX = toxicity state includes days with severe and life-threatening adverse events prior to progression only; TWiST = time without symptoms or toxicity; REL = relapse period until death or end of follow-up.

patients had advanced or metastatic HER $2+$ breast cancer and had progressive disease following prior therapy, which included an anthracycline (97\%), a taxane (97\%) and/or trastuzumab (97\%) Almost all patients had metastatic disease (96\%), with $78 \%$ involving visceral lesions and $49 \%$ having lesions at three or more sites.

During the entire study period, most patients experienced at least one $\mathrm{AE}(96 \%$ in the lapatinib plus capecitabine group and $89 \%$ in the capecitabine only group). In the combination group, $38 \%$ (76 of 198) of patients experienced a Grade 3/4 AE prior to progression or censoring for progression, with an average start time of 67 days after randomisation. In the capecitabine only group, the percentage was $36 \%$ (73 of 201), starting on average within 55 days of randomisation.

Figure 1 shows the unweighted mean duration of each health state. Overall median follow-up for survival was approximately 67 weeks. Average duration with Grade 3/4 AEs (prior to progression) was less than 2 weeks, and the difference between groups was not statistically significant. Mean time in the TWiST state (time without severe toxicities or symptoms of progression) comprised the primary difference in survival time between the treatment groups. The additional 3.5 weeks of overall survival for the combination group $v s$ the monotherapy group was more than accounted for by the difference in TWiST (32.1 weeks vs 21.3 weeks, $P<0.0001)$. Partitioned survival plots appear in Figure 2.

In the primary analysis, the difference in quality-adjusted survival time between combination and monotherapy ranged from 3 to 11 weeks (Table 1), depending on relative valuations of the health states. Differences were statistically significant except where REL was assumed to have a utility equal to TWiST. Thus, any patient who places less than full utility on time after progression is expected to gain more quality-adjusted survival with lapatinib, regardless of what value the patient places on the TOX state. When all states are valued at full utility (last row of Table 1), the mean survival advantage is 3.5 weeks $(P=0.1556)$ although the survival data is not yet fully mature. The threshold plot in Figure 3 illustrates the Q-TWiST differences between groups, with varying combinations of utility weights for TOX and REL.

As EQ-5D can produce negative utility values, we also ran the analyses using negative utilities for the TOX and REL states. All
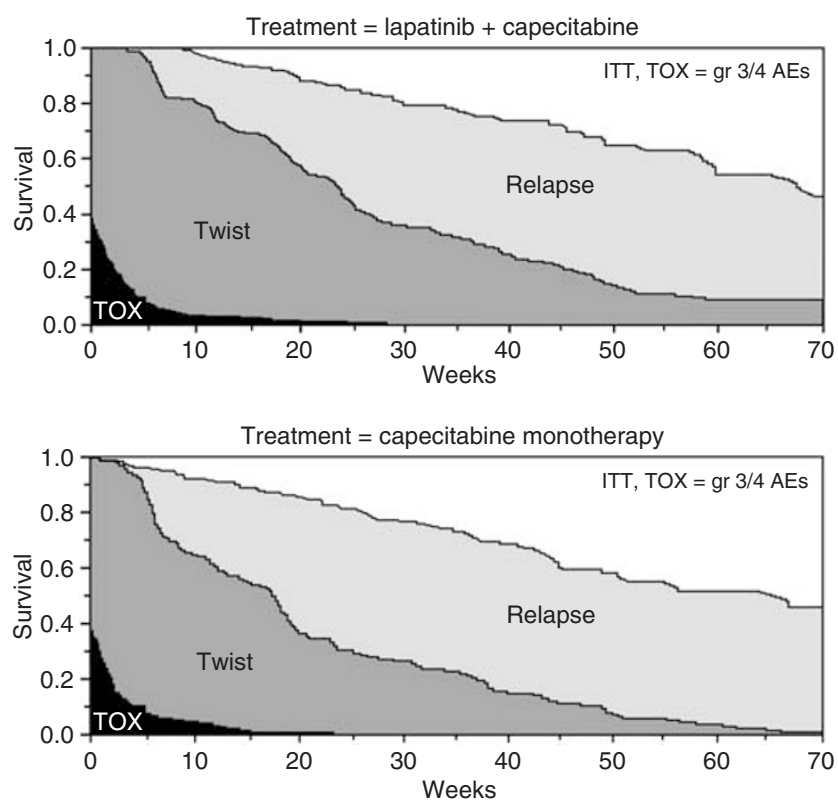

Figure 2 Partitioned survival curves. AEs=adverse events; $I T T=$ intentto-treat; $\mathrm{TOX}=$ toxicity; TWiST $=$ time without symptoms or toxicity

Table I Q-TWiST differences for varying combinations of utility weights

\begin{tabular}{|c|c|c|c|c|}
\hline \multicolumn{3}{|c|}{ Utility per health state } & \multicolumn{2}{|c|}{ Expected difference in Q-TWIST } \\
\hline Tox & TWiST & REL & (Weeks) & $P$-value \\
\hline 0 & । & 0 & 10.8 & 0.0000 \\
\hline 0.25 & 1 & 0 & 10.9 & 0.0000 \\
\hline 0.5 & i & 0 & 11.0 & 0.0000 \\
\hline 0.75 & 1 & 0 & 11.0 & 0.0000 \\
\hline 1 & 1 & 0 & 11.1 & 0.0000 \\
\hline 0 & i & 0.25 & 8.9 & 0.0002 \\
\hline 0.25 & । & 0.25 & 9.0 & 0.0001 \\
\hline 0.5 & i & 0.25 & 9.1 & 0.0001 \\
\hline 0.75 & i & 0.25 & 9.1 & 0.0001 \\
\hline 1 & I & 0.25 & 9.2 & 0.0001 \\
\hline 0 & i & 0.5 & 7.0 & 0.0017 \\
\hline 0.25 & 1 & 0.5 & 7.1 & 0.0015 \\
\hline 0.5 & i & 0.5 & 7.2 & 0.0013 \\
\hline 0.75 & i & 0.5 & 7.2 & 0.0012 \\
\hline 1 & 1 & 0.5 & 7.3 & 0.0012 \\
\hline 0.90 & i & 0.65 & 6.1 & 0.006 \\
\hline 0 & 1 & 0.75 & 5.1 & 0.0239 \\
\hline 0.25 & i & 0.75 & 5.2 & 0.0217 \\
\hline 0.5 & 1 & 0.75 & 5.2 & 0.0199 \\
\hline 0.75 & 1 & 0.75 & 5.3 & 0.0185 \\
\hline I & i & 0.75 & 5.4 & 0.0175 \\
\hline 0 & I & 1 & 3.2 & 0.1912 \\
\hline 0.25 & i & i & 3.3 & 0.1807 \\
\hline 0.5 & 1 & 1 & 3.3 & 0.1712 \\
\hline 0.75 & i & I & 3.4 & 0.1629 \\
\hline 1 & i & I & 3.5 & 0.1556 \\
\hline
\end{tabular}

Q-TWiST = quality-adjusted time without symptoms of disease or toxicity of treatment. Positive values indicate longer duration for combination therapy than monotherapy. Highlighted row shows results using average observed utility values relative to TWiST.

comparisons resulted in a positive Q-TWiST advantage for the $\mathrm{L}+\mathrm{C}$ combination vs $\mathrm{C}$ monotherapy. Negative utilities on the TOX state diminished the advantage, whereas negative utilities on the REL state increased the advantage. In other words, the less 
combinations. Most differences were statistically significant between groups. Differences were less pronounced only if REL was valued at the level of TWiST or if REL was valued almost at the level of TWiST and higher than TOX.

Patient-reported utility data from the trial was used to estimate average utility weights for the health states. However, there are limitations on basing quality-adjusted survival on actual patient experience of the treatments administered. EQ-5D data were not reported on all patients or at all visits, and fewer than half of the patients had an end-of-study assessment. Furthermore, to use EQ-5D data to ascribe utilities to the TOX state, the assessment should have taken place on a day when the $\mathrm{AE}$ was being experienced, and this was uncommon. REL utilities were based on values collected at withdrawal from study treatment and could be determined only for patients who experienced the progression event, not for those who were censored for the event.

Despite these limitations, the overall average reported utility for REL was 0.43, which is in the 0.41-0.69 range reported by Earle et al (2000) for progressive metastatic breast cancer. At this level, and with average utility for TOX and TWiST at 0.59 and 0.66 , respectively, the lapatinib plus capecitabine combination treatment used in this study provides, on average, significantly more quality-adjusted survival time than does treatment with capecitabine alone.

Earle et al (2000) also report utility in the range of $0.16-0.54$ for terminal metastatic breast cancer, and Nooij et al (2003) cite a

\section{REFERENCES}

Agency for Healthcare Research and Quality (2005) Calculating the US Population-Based EQ-5D Index Score. Rockville, MD, Available at: http://www.ahrq.gov/rice/EQ5Dscore.htm Accessed 17 October 2006

Cameron D, Casey M, Press M, Lindquist D, Pienkowski T, Romieu CG, Chan S, Jagiello-Gruszfeld A, Kaufman B, Crown J, Chan A, Campone M, Viens P, Davidson N, Gorbounova V, Raats JI, Skarlos D, Newstat B, Roychowdhury D, Paoletti P, Oliva C, Rubin S, Stein S, Geyer CE (2008) A phase III randomized comparison of lapatinib plus capecitabine versus capecitabine alone in women with advanced breast cancer that has progressed on trastuzumab: updated efficacy and biomarker analyses. Breast Cancer Res Treat; e-pub ahead of print

Cole BF, Gelber RD, Gelber S, Coates AS, Goldhirsch A (2001) Polychemotherapy for early breast cancer: an overview of the randomised clinical trials with quality-adjusted survival analysis. Lancet 358: 277-286

Earle CC, Chapman RH, Baker CS, Bell CM, Stone PW, Sandberg EA, Neumann PJ (2000) Systematic overview of cost-utility assessments in oncology. J Clin Oncol 18: 3302-3317

EuroQol Group (1990) EuroQol-a new facility for the measurement of health-related quality of life. Health Policy 16: 199-208

Gelber RD, Goldhirsch A (1986) A new endpoint for the assessment of adjuvant therapy in postmenopausal women with operable breast cancer. J Clin Oncol 4: 1772-1779 physician-based utility $=0.23$ for the progressive state in metastatic breast cancer. These lower values may reflect a more realistic range of values than the observed values for the entire period after progression, as the deterioration in health that eventually occurs may not be captured well by assessments that were taken close to the progression date. Application of lower utilities for the REL state would result in a more pronounced Q-TWiST advantage for the lapatinib plus capecitabine treatment combination.

In summary, the lapatinib plus capecitabine combination provided significantly greater Q-TWiST than did capecitabine alone. The full impact of the combination cannot be determined, because of the early closure to accrual and subsequent cross over, but it is likely that the average 7 weeks improvement is an underestimate of the overall benefit.

\section{ACKNOWLEDGEMENTS}

We thank the patients who participated in the study on which this analysis is based and their families; the medical, nursing and research staff at the study centres; the independent data and safety monitoring committee; the monitors, clinical operations staff, data managers, statisticians, and programmers at GlaxoSmithKline. The study and the Q-TWiST analysis reported in this paper were funded by GlaxoSmithKline.
Gelber RD, Goldhirsch A, Cavalli F (1991) Quality-of-life-adjusted evaluation of adjuvant therapies for operable breast cancer. The International Breast Cancer Study Group. Ann Intern Med 114: $621-628$

Geyer CE, Forster J, Lindquist D, Chan S, Romieu CG, Pienkowski T, Jagiello-Gruszfeld A, Crown J, Chan A, Kaufman B, Skarlos D, Campone M, Davidson N, Berger M, Oliva C, Rubin SD, Stein S, Cameron D (2006) Lapatinib plus capecitabine for HER2-positive advanced breast cancer. N Engl J Med 355: 2733-2743

Glasziou PP, Simes RJ, Gelber RD (1990) Quality adjusted survival analysis. Stat Med 9: 1259-1276

Kaplan EL, Meier P (1958) Nonparametric estimation from incomplete observations. J Am Stat Assoc 53: 457-481

Nooij MA, de Haes JC, Beex LV, Wildiers J, Klijn J, Becquart D, Jassem J, Engelsman E, Duchateau L, EORTC Breast Cancer Group (2003) Continuing chemotherapy or not after the induction treatment in advanced breast cancer patients. Clinical outcomes and oncologists' preferences. Eur J Cancer 39: 614-621

Revicki DA, Feeny D, Hunt TL, Cole BF (2006) Analyzing oncology clinical trial data using the Q-TWiST method: clinical importance and sources for health state preference data. Qual Life Res 15: $411-423$ 